\title{
Basal Implant: A Remedy to Restore Resorbed Alveolar Ridges
}

\author{
Prakhar Thakur $^{1} \quad$ Tarun Kalra $^{1} \quad$ Manjit Kumar $^{1} \quad$ Ajay Bansal $^{1} \quad$ Shefali Malik ${ }^{1}$ \\ 1Department of Prosthodontics, Bhojia Dental College and Hospital, \\ Baddi District, Solan, Himachal Pradesh, India \\ Address for correspondence Manjit Kumar, MDS, Department of \\ Prosthodontics, Bhojia Dental College and Hospital, Baddi District, \\ Solan - 173205, Himachal Pradesh, India \\ (e-mail: manjitkiran@yahoo.co.in).
}

Dent J Adv Stud 2021;9:61-65.

\begin{abstract}
The conventional crestal implants are used only when there is adequate jawbone height and width. Results of conventional implants are good in patients with healthy bone at the time of treatment, but prognosis gets deteriorated when surgical augmentation of bone is included with implant placement. These augmentation procedures have surgical risks and are costlier to the patients. Patients with atrophied jawbones are given no treatment, until crestal implants are seen as the last option. In this article, the indications for basal implants and functional differences between basal implants and crestal implants have been discussed.

Patients with extreme jawbone atrophy do not benefit from crestal implants. The basal bone is the (cortical) osseous tissue of the mandible and maxilla, and lies below the alveolar process, which has a relatively strong and no resorbing framework.

Basal osseointegrated and basal cortical screw (BCS) are two types of implants designed to take anchorage from the cortical bone of the jaw. BCS implants have long shafts and can be placed immediately in the socket after extraction and provided with immediate loading within 72 hours of implant placement. Basal implants are also called bicortical

Keywords

- basal implants

- crestal implants

- immediate loading or cortical implants as they utilize the cortical portion of the jawbones for anchorage and implant stability. The basal bone has better quality and quantity of cortical bone for retention of these unique and highly advanced implants. The other names for these implants are lateral implants or disk implants.
\end{abstract}

\section{Introduction}

Academic researchers and dentists focus their treatment completely on crestal implants. Screw- and cylinder-type implants, which put the masticatory load on cancellous bone, draw the attention of the prosthodontists. When availability of bone is less, other methods such as distraction osteogenesis and guided bone growth, are used to rebuild the bone tissue by transplantation. ${ }^{1}$
If there is less bone, it is difficult to put crestal implants. These procedures are costly and time-consuming. Therefore, many patients cannot afford them and thus their masticatory function cannot be restored in a truly comprehensive manner. ${ }^{2}$

The implant technique of "basal osseointegration" (BOI) has been developed with a view to address the situations outlined earlier, among other problems. Modified techniques of lateral access to the jawbone should be the standard technique in

(C) 2021. Bhojia Dental College and Hospital affiliated to Himachal Pradesh University.

This is an open access article published by Thieme under the terms of the Creative Commons Attribution-NonDerivative-NonCommercial-License, permitting copying and reproduction so long as the original work is given appropriate credit. Contents may not be used for commercial purposes, or adapted, remixed, transformed or built upon. (https://creativecommons.org/licenses/by-nc-nd/4.0/).

Thieme Medical and Scientific Publishers Pvt. Ltd. A-12, 2nd Floor, Sector 2, Noida-201301 UP, India 
dental implantology. Conventional implant designs can occasionally be used as additional treatment options.

Even though a sustainable implant site might be unusable at the time of first implantation, the treatment can still be planned in such manner that the specified bone volume is built up under a long-term temporary restoration. The basal implant has the capability to mineralize the remaining cortical bone in the jaw.

There are situations where the mineralization pattern not consistent with what is expected or where adequate bone volume is absent at strategically favorable implant positions. This may occur at the beginning of or during a treatment. ${ }^{3,4}$

In such cases, stability is not achieved during the initial phase of treatment. However, with the use of basal implants, enhanced mineralization of the bone and stability can be achieved with progressive loading during bone healing.

\section{Characteristics of Basal Implant}

Crestal implantology no doubt offers optimal ways of restorative treatment when the health of the available bone is ideal. These criteria are not met by most of the patients. This is particularly true of the posterior segments of the maxilla and mandible. Whenever oral conditions are not ideal for crestal implants, the benefits of the BOI approach are obvious:

- Low degree of invasiveness (no augmentation, distraction, or transplantation)

- One-step procedure

- Simple repair in case of problems

- Combination with natural teeth possible

- Combination with crestal implants possible

- Manageable system (few components)

- Simple laboratory technique

- Extremely high success rates

Presumably, prognosis of the BOI implants is better from the start, as the blood supply to the bone remains largely intact in the area where implant is placed inside the basal (cortical) bone.

Crestal implants with modified surface characteristics can lead to peri-implant inflammation, which can lead to ridge resorption. This is less in the case of the BOI technique because of its narrow emergence profile. The bacterial invasion is far away from the site of force of mastication. So an additional advantage is bone not being exposed to two tasks of inflammation and occlusion at the same time.

The cortical bone uses osteogenic and osteoprotective properties as the implant is osteointegrated. In BOI, the design is such that the masticatory surface and load transmission are far from each other, so the health of the alveolar process is not affected.

For successful insertion and force transmission, it is irrelevant whether the vertical portion of the implant is embedded in air or in fluid. It is also irrelevant whether bone tissue is deposited directly on the implant surface during this area. This has many desirable implications. Aesthetic considerations aside, the success of implant therapy does not depend upon the presence of alveolar bone.
As is well known, crestal implants are inserted from the crest of the alveolar bone and are indicated in the cases where sufficient bone height is available. Their success decreases as available bone decreases Also, augmentation procedures increase overall cost of implant treatment. Crestal implants are not indicated in patients with severely atrophied jawbones.

The maxilla and the mandible constitute the basal bone under the alveolar process, which remains unchanged. Smoking does not affect the basal implants. These are of two types of implants: BOI and basal cortical screw (BCS) implants. They are indicated and designed for implantation in the cortical bone of the jaw. The screwable basal implants are long and are indicated for immediate extraction socket.

These are known as bicortical or cortical implants as they use the cortical bone for retention. The basal bone has excellent quality and quantity of cortical bone for retention. These implants are also called lateral implants or disk implants.

\section{Indications}

- All situations of freshly extracted and missing teeth.

- When bone augmentation and two-stage implants have failed. ${ }^{5,6}$

- All kinds of bone atrophy that includes very thin ridges, buccolingually, and insufficient bone height.

These situations develop due to prolong use of removable dentures, which lead to resorption of bone and height reduction. Prong edentulism, untreated periodontal disease (diabetics), and trauma to the jaw may damage and cause resorption of the alveolar bone.

\section{Contraindications}

- Special cases: Cases in which the muscles and ligaments are deficient and balanced occlusion could not be achieved and immediate loading cannot be done.

- Medical conditions: Dental implants are contraindicated in many medical conditions, such as cardiac patients with myocardial infarction or cerebrovascular accident and immunosuppressant.

- Medicines: A dentist will need information about the patient's intake of medicines and supplements, for example, drugs involved in treatment of cancer, anticoagulants, and bisphosphonates (for osteoporosis).

\section{Advantages of Basal Implants}

- Immediate loading: It saves time as implants are loaded with prosthesis within 72 hours and cost of implant is also low. It takes 6 months to a year in the cases where bone or grafting procedures are adopted. The provisional prosthesis is not required in these implants. ${ }^{7}$

- One-piece implantology: There is less chance of failure of implants because there is no implant abutment interface as in conventional implants.

- Basal-cortical bone support: Support is being taken from the basal bone in these implants from the basal bone, 
which resorbs very less, is more stable, and is fast repairable as compared to implants that take support from the crestal bone.

- Less invasive, minimal surgical complications: One-piece implants are done without raising the flap, so it is less invasive with less edema and less postoperative complications.

- Compromised bone situations: In the cases where there is less bone, augmentation/grafting and bone substitutes are used. But in basal implants, as the cortical bone is used, such procedures are not needed.

- Distribution of masticatory forces: These implants use the cortical bone for retention, which resorbs less, has a fast repairable quality, and can take better masticatory load. ${ }^{8,9}$

- Peri-implantitis incidence: Inflammation around implants is a very common cause of failure in implants. This happens due to the rough surface of the conventional implant. Because of smooth surface and monoblock basal implant, there is up to $98 \%$ less peri-implantitis in basal implants.

- Medically compromised situations: These implants can be used in patients with diabetes, smoking habit, and periodontitis.

- Diabetes mellitus: Wound healing is delayed and there are more chances of gingivitis and periodontitis in diabetic patients, so normal implants are contraindicated. But basal implants can be given in controlled diabetic patients, because they have a smooth implant surface and, therefore, there is less deposition of bacteria and less chance of disease.

- Smoking: Because of chronic irritation of nicotine and high temperature rise, there is less blood flow in the wound site and healing is delayed, which leads to complications. There are more chances of gingivitis and gingival recession, which lead to inflammation around the implant and thus implant failure. In the cases of basal implants, the masticatory load area is far away from the smoking zone, so there is better osseointegration. However, smoking is definitely contraindicated during the healing period of an implant surgery.

- Acute destructive periodontitis: In acute periodontitis, there are chances of infections leading to failure of implants. There may be more mobility of teeth and painful and inflamed gums. In these cases, conventional implants are contraindicated. However, because of the smooth implant surface, there are less chances of deposition of bacteria and thus less chances of diseases. ${ }^{10}$

- Cost savings: Because bone grafts and second-stage surgery are not required, treatment time is reduced, which helps in saving costs.

\section{Disadvantages of Basal Implants}

For insertion of BOI implants, open flap surgery is needed. mucosal incision and later on closure by sutures are required as compared to BCS implants, in which implants are inserted without raising a flap. For placement of these implants, high-skilled and experienced prosthodontists are required to avoid complications. ${ }^{11}$

\section{Types of Implants}

These are of two types if implants: BOI and BCS. Implants have plates and threads for better osseointegration. Screws with a diameter of up to $12 \mathrm{~mm}$ are available and are indicated for fresh extraction socket.

\section{Lateral Basal Implants}

These implants are inserted from the lateral side of the jawbones. The masticatory forces are exerted in the horizontal direction within the cortical bone structures ( - Fig. 1).

\section{Anterior Implants}

These implants have two disks: the basal disk (9 to $10 \mathrm{~mm}$ diameter) and crestal disk ( $7 \mathrm{~mm}$ diameter). Sufficient vertical space is needed in these cases. The disks provide different functions. The crestal plate provides additional initial stability. Later on, the basal plate becomes osseointegrated. If there is deficient bone, single-disk BOI with a 7 to $9 \mathrm{~mm}$ diameter and shafts between 8 and $13.5 \mathrm{~mm}$ can be used.

\section{Posterior Implants}

The posterior implants are of a square shape, with a disk of 9 to 12 or 10 to $14 \mathrm{~mm}$, with shaft lengths of 10 to $13.5 \mathrm{~mm}$, according to the available horizontal bone. The infra-nerve implant insertion in the form of a disk can be made below the mandibular nerve and the thread carrier is found at the lateral surface of the nerve where bone height is less than $2 \mathrm{~mm}$ above the nerve.

\section{Screw Basal Implant}

Screw-shaped implants are inserted through the mucosa without incision like in conventional implants. Bicortical screws are also considered basal implants, as masticatory forces are transmitted without osseointegration. ${ }^{12}$ Bicortical screws show some elasticity; since they have a polished surface and are less invasive, there are less chances of inflammation ( - Fig. 2 ).

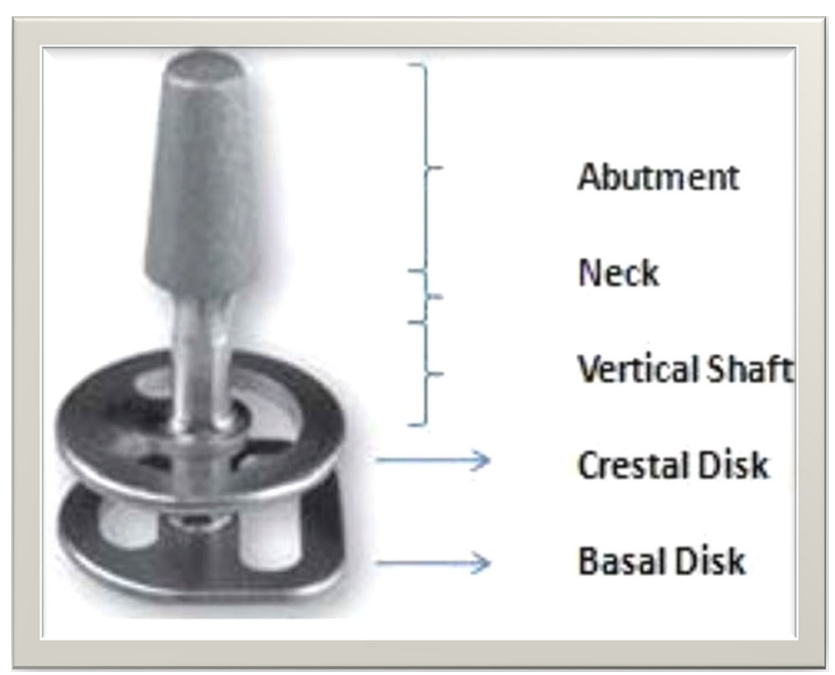

Fig. 1 Disk implant. 


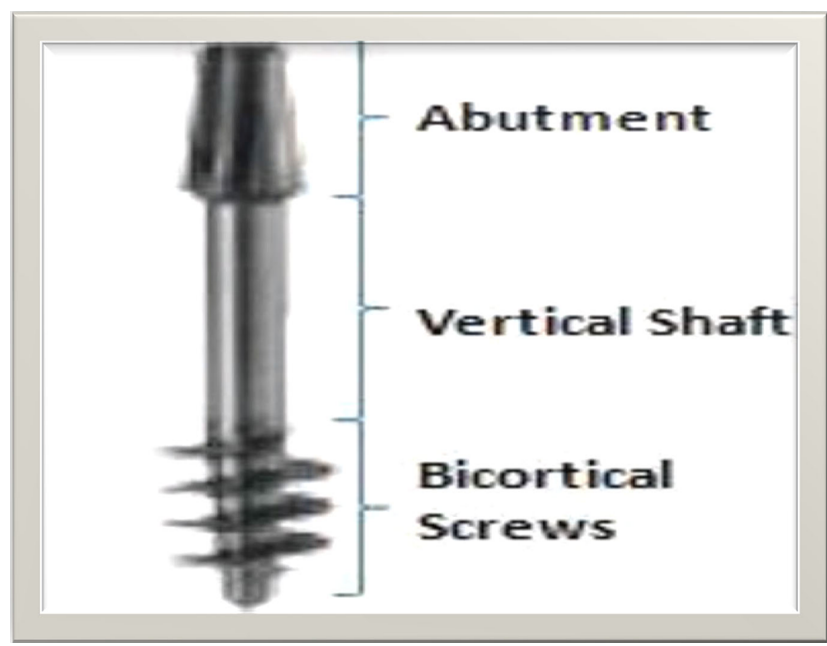

Fig. 2 Basal cortical screw (BCS) implant.

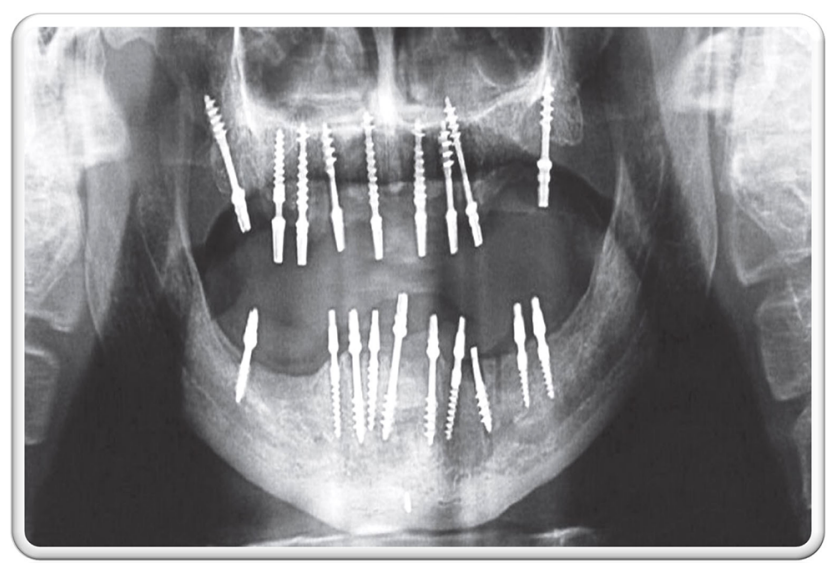

Fig. 4 Orthopantograph showing full mouth rehabilitation with basal implant.

\section{Parts of Basal Implants}

These are single-piece implants without an implant-abutment interface, which leads to less failure as seen in conventional implants.

\section{Implant Surface}

Because they have a polished surface, there are less chances of deposition of bacterial plaque.

\section{Implant Body}

Implant body is thin with wide threads, which enhances vascularity around implant and increases bone-implant contact.

\section{Implant Neck}

As the implant is placed in dense corticated bone and depending on length of the implant, the angled abutment can be up to 15 to 25 degrees.

\section{Summary}

When we need to restore edentulous ridges with ideal width and height, conventional implants are often placed that

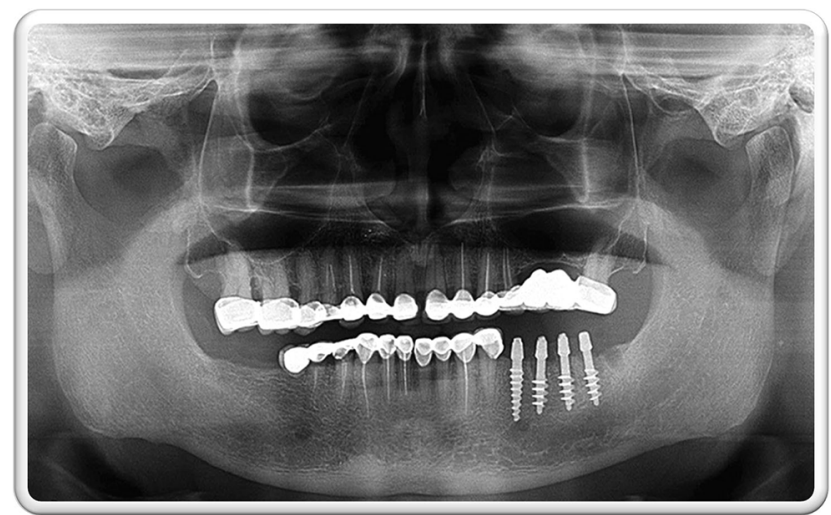

Fig. 3 Orthopantograph showing immediate basal implant placement.

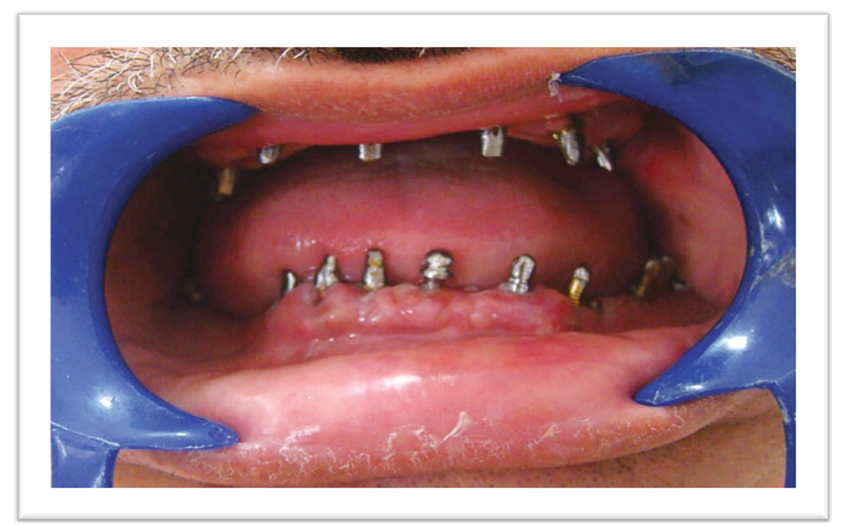

Fig. 5 Intraoral view of full mouth rehabilitation with basal implant.

osseointegrate inside the trabecular bone. For atrophic ridges, we cannot place these conventional implants as there is significant loss of trabecular bone. In these cases, we will place basal implants within the basal bone. This bone is a tough cortical bone below the alveolar bone and does not undergo resorption (-Figs. 3-5). Basal implants are accustomed to support single- and multiple-unit restorations within both jaws. These implants can be inserted within the extraction sockets and in healed bones. For resorbed ridges, we will use basal implants. These implants do not require bone grafting and augmentation procedures, thus saving treatment time. Immediate loading is completed in 72 hours and thus provides psychological relief to patients.

\section{Conflict of Interest}

None declared.

\section{Source of Funding}

None.

\section{References}

1 Yadav RS, Sangur R, Mahajan T, Rajanikant AV, Singh N, Singh R. An alternative to conventional dental implants: basal implants. Rama Univ J Dent Sci 2015;2(2):22-28

2 Stefan I. Comparison of basal and crestal implants and their modus of application. Smile Dent J 2009;4(1):36-46 
3 Ihde S, Ihde A, Lysenko V, Konstantinovic V, Palka L. New systematic terminology of cortical bone areas for osseo-fixated implants in strategic oral implantology.JJ Anatomy 2016;1:1-7

4 Kopp S. Basal implants: a safe and effective treatment option in dental implantology. CMF Impl Dir 2007;3:110-115

5 Stefan I. Immediate restoration after failure and replacement of basal implants. CMF Impl Dir 2008;3:28-34

6 Ihde S, Goldmann T, Himmlova L, Aleksic Z. The use of finite element analysis to model bone-implant contact with basal implants. Oral Surg Oral Med Oral Pathol Oral Radiol Endod 2008;106(1):39-48

7 Diedrich $\mathrm{H}$. Immediate loading of a maxillary full-arch rehabilitation supported by basal and crestal implants. Craniomaxillofacial Implant Directions 2008;1:61-63

8 Goldmann T, Ihde S, Kuzelka J, Himmlova L. Bendable vs. angulated dental implants: consideration of elastic and plastic material properties based on experimental implant material data and FEA. Biomed Pap Med Fac Univ Palacky Olomouc Czech Repub 2008;152(2):309-316

9 Kopp S, Kuzelka J, Goldmann T, Himmlova L, Ihde S. Modeling of load transmission and distribution of deformation energy before and after healing of basal dental implants in the human mandible. Biomed Tech (Berl) 2011;56(1):53-58

10 Sigmar K. "All on four": basal implants as solid base for circular bridges in high periodontal risk patients. CMFImpl Dir 2007;3:105-118

11 Nair C, Bharathi S, Jawade R, Jain M. Basal implants: a panacea for atrophic ridges. Journal of Dental Sciences and Oral Rehabilitation 2013;1:1-4

12 Narang S, Narang A, Jain K, Bhatia V. Multiple immediate implants placement with immediate loading. J Indian Soc Periodontol 2014;18(5):648-650 